\title{
Bayesian Estimation for Exponentiated Gamma Distribution Under Progressive Type-II Censoring Using Different Approximation Techniques
}

\author{
Sanjay Kumar Singh1, Umesh Singh 1, Abhimanyu Singh Yadav2* \\ Department of Statistics and DST-CIMS, Banaras Hindu University Varanasi \\ Department of Statistics, PUC, Mizoram University, Aizawl,
}

\begin{abstract}
In this paper, we proposed the Bayesian estimation for the parameter and reliability function of exponentiated gamma distribution under progressive type-II censored samples. The Bayes estimate of the parameter and reliability function are derived under the assumption of independent gamma prior by three different approximation methods namely Lindley's approximation, Tierney-Kadane and Markov Chain Monte Carlo methods. Further, the comparison of Bayes estimators with corresponding maximum likelihood estimators have been carried out through simulation study. Finally, a real data set has been used to illustrate the above study in realistic phenomenon.
\end{abstract}

Keywords:Exponentiated Gamma distribution, Progressive Censoring, Lindley's approximation, T-K approximation, MCMC technique.

\section{Introduction}

In survival and clinical trial studies exponential distribution is the most widely used for data analysis and is preferred for the situations where failure rate is constant in nature. In case of monotonic failure rate, a number of distributions have been suggested but Weibull and gamma distributions are most frequently used. The gamma distribution has a major constraint that its survival function cannot be expressed in nice closed forms, thus, it create difficulties for further mathematical modifications. The survival and hazard function for the such distribution are often evaluated numerically. This is one of the important reasons that made the gamma distribution unpopular in comparison to the Weibull distribution. Although Weibull distribution has a nice closed form for hazard and survival function, but it has its own disadvantages. Gupta et al. (1998) have proposed the use of exponentiated gamma distribution as an alternative to gamma and Weibull distributions. Shawky and Bakoban (2008) have discussed the applicability of exponentiated gamma distribution and derived Bayesian and non-Bayesian estimators of the shape parameter, reliability 
and failure rate functions in the case of complete and type-II censored samples. Further, Shawky and Bakoban (2009) considered the order statistics from an exponentiated gamma (EG) distribution and discussed the several mathematical properties based on order statistics. Singh et al. (2011), Ghanizadeh et al. (2011) and Khan and Kumar (2011) have also proposed the estimation procedure for the EGD under complete and censored data.

The probability density function and reliability function of exponentiated gamma distribution (EGD) are given by following equations;

$$
f(x, \alpha)=\alpha x e^{-x}\left\{1-e^{-x}(1+x)\right\}^{\alpha-1} \quad ; x \geq 0, \alpha>0
$$

and

$$
R(t)=1-\left\{1-e^{-t}(1+t)\right\}^{\alpha} \quad ; t, \alpha>0
$$

It may be noted here that, the EGD is a simple generalization of the Gamma distribution with known shape and scale parameters, namely $\mathrm{G}(2,1)$. This distribution is parsimonious in parameters and hence, simple to use. The other advantage is that, it has various shapes of hazard function for different values of the shape parameter $\alpha$. It has increasing hazard function when $\alpha>1 / 2$ and takes Bath-tub shape for $\alpha<1 / 2$. For other details about this distribution, we refer Shawky and Bakoban (2008), Singh et al. (2011).

\subsection{Progressive Censoring and Likelihood Function}

In a typical life testing experiments, Type-I and Type-II censoring schemes are the two most popular schemes. Both schemes have its own advantages and disadvantages. Usually, life testing experiments are costly and time consuming. Therefore, the experimenter terminate the experiment at a prefixed time $\mathrm{T}$ or prechosen number of failure $\mathrm{R}$. Therefore, in Type-I censoring scheme, censoring time $\mathrm{T}$ is prefixed and number of failure $\mathrm{R}$ is random, while in Type-II censoring scheme number of failure $\mathrm{R}$ is prefixed and termination time $\mathrm{T}$ of the experiments is random. The above discussed censoring schemes does not provide the flexibility of removing the experimental units during the experiment time. Therefore, more generalized version of the Type-II censoring schemes is proposed by Cohen (1965) named as progressive Type-II censoring (PT-II) scheme. PT-II censoring provides the facility of removals of the units during the experiments and reduces the cost and experiment time. This may be the reason behind its popularity. For more detail about the PT-II censoring readers may refer to Balakrishana and Aggarwala (2000), Balakrishana and Hossain (2007), Balakrishnan and Shandhu (1965), Krishna and Kumar (2011).

In case of PT-II censoring scheme, the likelihood function is given by the following equations. 


$$
L(x \mid \alpha, R)=\psi \prod_{j=1}^{m} f\left(x_{j}\right)\left\{1-F\left(x_{j}\right)\right\}^{R_{j}}
$$

where, $\psi=n\left(n-R_{1}-1\right), \cdots,\left(n-R_{1}-R_{2}-\cdots-R_{m-1}-m+1\right)$. Therefore, the likelihood function using (1) can be written as;

$L(x \mid \alpha, R) \propto \alpha^{m} e^{-\sum_{j=1}^{m} x_{j}} \prod_{j=1}^{m}\left[x_{j}\left\{1-e^{-x_{j}}\left(1+x_{j}\right)\right\}^{\alpha-1}\left\{1-\left\{1-e^{-x_{j}}\left(1+x_{j}\right)\right\}^{\alpha}\right\}^{R_{j}}\right]$

Now, the log-likelihood function is given by

$$
\begin{array}{r}
\ln L \propto m \ln \alpha-\sum_{j=1}^{m} x_{j}+\sum_{j=1}^{m} \ln x_{j}+(\alpha-1) \sum_{j=1}^{m}\left[1-\left(1+x_{j} e^{-x_{j}}\right)\right] \\
+\sum_{j=1}^{m} R_{j} \ln \left\{1-\left\{1-e^{-x_{j}}\left(1+x_{j}\right)\right\}^{\alpha}\right\}
\end{array}
$$

The layout of this paper is as follows; In Section 1, we provides the basic introduction and literatures related to the considered model. The MLEs are obtained in Section 2. The Bayes procedure for EGD under different approximation techniques are discussed in Section and Subsections of 3. The real data analysis and numerical comparison are presented in Sections 4 and 5 respectively. Finally, in Section 6, we conclude the paper.

\section{Maximum Likelihood Estimation}

The maximum likelihood estimate (MLE) of the parameter is obtained by using the above equation (5). To obtain MLE, firstly we differentiate the log-likelihood equation w.r.t. the parameter and equate it to zero. Thus, we have;

$$
\frac{\partial \ln L}{\partial \alpha}=\frac{m}{\alpha}+\sum_{j=1}^{m} \ln \phi_{x_{j}}-\sum_{j=1}^{m} R_{j} \frac{\phi_{x_{j}}^{\alpha} \ln \left(1-\phi_{x_{j}}\right)}{\left(1-\phi_{x_{j}}^{\alpha}\right)}=0
$$

where, $\varphi_{x j}=\left[1-\left(1+x_{j} e^{-x j}\right)\right]$. Therefore, MLE of $\alpha$ is the solution of the above equation (6). But we observed that, the analytical solution of the above equation is not possible. Thus, we proposed the use of Newton-Raphson (N-R) algorithm 
554 Bayesian Estimation for Exponentiated Gamma Distribution Under Progressive Type-II Censoring Using Different Approximation Techniques

\subsection{Asymptotic confidence interval}

to calculate MLE. Further, if we suppose that ${ }^{\wedge} \alpha \mathrm{M}$ is the MLE of $\alpha$. Then, the MLE of the reliability function is obtained by using the invariance property of MLE which is given in following equation.

$$
\hat{R(t)})_{M}=\left[1-\left\{1-e^{-t}(1+t)\right\}^{\hat{\alpha}_{M}}\right]
$$

\subsection{Asymptotic confidence interval}

To construct asymptotic confidence interval, we need to obtain the observed Fisher information. Therefore, the Fisher information matrix is given by

$$
I(\hat{\alpha})=-E\left(\frac{\partial^{2} L}{\partial \alpha^{2}}\right)_{\alpha=\hat{\alpha}}
$$

also the asymptotic variance of $\alpha$ is given by

$$
\operatorname{Var}(\hat{\alpha})=\frac{1}{I(\hat{\alpha})}
$$

But, the exact mathematical expressions for the above expectation is not exist. Therefore, by using the concept of large sample theory the $100(1-\varphi) \%$ confidence interval of $\alpha$ is given by;

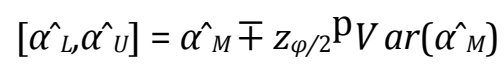

\section{Bayesian Estimation}

In this section, we have obtained the Bayes estimates for the unknown parameters $\alpha$ and reliability function $\mathrm{R}(\mathrm{t})$ based on PT-II censored data. In Bayesian analysis, the parameter of interest is to be considered as a random variable and follows some prior distribution. Here, we assume that, parameter $\alpha \sim$ gamma(a,b) density i.e.

$$
\pi(\alpha) \propto \alpha a-1 e-b \alpha ; \alpha>0
$$

where $\mathrm{a}, \mathrm{b}$ are the hyper parameters which are assumed known. The above considered prior is more applicable in the sense that, it is more flexible and assumes different variety of prior which may be the reason behind its popularity. To obtain the Bayes estimates these quantities, squared error loss function is taken and defined as;Squared error loss function $(\operatorname{SELF}): \operatorname{LS}\left(\alpha, \alpha^{\wedge}\right)=\left(\alpha^{\wedge}-\alpha\right) 2$, 
In each case ${ }^{\wedge} \alpha$ represents the estimates of unknown parameter $\alpha$. The Bayes estimate of $\alpha$ with respect to the LS loss function is obtained from its posterior distribution as;

$$
\hat{\alpha} S=\left\{E_{\alpha}(\alpha \mid \underline{\mathrm{x}})\right\}
$$

\subsection{Lindley's Approximation Method provided}

the above expectation exist.

Therefore, based on the above prior, the posterior distribution of $\alpha$ is given

as;

$$
\begin{aligned}
& p(\alpha \mid \underline{\mathrm{x}}) \propto \prod_{j=1}^{m}\left[x_{j}\left\{1-e^{-x_{j}}\left(1+x_{j}\right)\right\}^{\alpha-1}\left\{1-\left\{1-e^{-x_{j}}\left(1+x_{j}\right)\right\}^{\alpha}\right\}^{R_{j}}\right] \\
& \alpha^{m+a-1}\left\{e^{-\sum_{j=1}^{m} x_{j}-b \alpha}\right\}
\end{aligned}
$$

Now, the Bayes estimators of the parameter and reliability function under SELF is given as;

$$
\hat{\alpha}_{B}=\frac{\int_{\alpha=0}^{\infty} \alpha^{m+a}\left\{e^{-\sum_{j=1}^{m} x_{j}-b \alpha}\right\} \mu\left(\alpha, x_{j}\right) d \alpha}{\int_{\alpha=0}^{\infty} \alpha^{m+a-1}\left\{e^{-\sum_{j=1}^{m} x_{j}-b \alpha}\right\} \mu\left(\alpha, x_{j}\right) d \alpha}
$$

and

$$
\hat{R}_{B}=\frac{\int_{\alpha=0}^{\infty}\left[1-\left\{1-e^{-t}(1+t)\right\}^{\alpha}\right] \alpha^{m+a-1}\left\{e^{-\sum_{j=1}^{m} x_{j}-b \alpha}\right\} \mu\left(\alpha, x_{j}\right) d \alpha}{\int_{\alpha=0}^{\infty} \alpha^{m+a-1}\left\{e^{-\sum_{j=1}^{m} x_{j}-b \alpha}\right\} \mu\left(\alpha, x_{j}\right) d \alpha}
$$

where, $\mu\left(\alpha, x_{j}\right)=\prod_{j=1}^{m}\left[x_{j}\left\{1-e^{-x_{j}}(1+x j)\right\}^{\alpha-1}\left\{1-\left\{1-e^{-x j}(1+x j)\right\}^{\alpha}\right\}^{R j}\right]$

From the above equations we observed that, the analytical solution of the integrals $(9,10)$ is not possible due to the implicit mathematical form and thus, we require some approximation techniques. Therefore, here the authors are proposing the use of three different approximation method namely 
556 Bayesian Estimation for Exponentiated Gamma Distribution Under Progressive Type-II Censoring Using Different Approximation Techniques

Lindley's approximation method, T-K approximation and Markov Chain Monte Carlo method for evaluation the Bayes estimates of the parameter and reliability function.

\subsection{Lindley's Approximation Method}

In the previous section, we obtained Bayes estimates based on PT-II censored data observed from EGD. These estimators are derived against squared error loss

\subsection{T-K Approximation Method}

functions. It is easy to observed that all these estimates are in the form of ratio of two integrals for which simplified closed forms are not available. Thus, one can apply Lindley's (1980) approximation methods to evaluate these estimates. Using this method the Bayes estimates are obtained by following equations;

$$
\hat{\alpha}_{B L}=\hat{\alpha}_{M}+\hat{\tau}_{\alpha} \hat{\sigma}_{\alpha \alpha}+\frac{1}{2} \hat{\sigma}_{\alpha \alpha}^{2} \hat{L}_{\alpha \alpha \alpha}
$$

and

$$
\hat{R}_{B L}=\hat{R}_{M}+\frac{1}{2} \hat{r}_{\alpha \alpha} \hat{\sigma}_{\alpha \alpha}+\hat{r}_{\alpha} \hat{\tau}_{\alpha} \hat{\sigma}_{\alpha \alpha}+\frac{1}{2} \hat{r}_{\alpha} \hat{\sigma}_{\alpha \alpha}^{2} \hat{L}_{\alpha \alpha \alpha}
$$

where,

$$
\begin{gathered}
\frac{\partial^{2} L}{\partial \alpha^{2}}=L_{\alpha \alpha}=-\frac{m}{\alpha^{2}}-\sum_{i=1}^{m} \frac{R_{i}\left[1-e^{-x_{i}}\left(1+x_{i}\right)\right]^{\alpha}\left\{\ln \left[1-e^{-x_{i}}\left(1+x_{i}\right)\right]\right\}^{2}}{\left(1-\left[1-e^{-x_{i}}\left(1+x_{i}\right)\right]^{\alpha}\right)^{2}} \\
\frac{\partial^{3} L}{\partial \alpha^{3}}=L_{\alpha \alpha \alpha}=\frac{2 m}{\alpha^{3}}-\sum_{i=1}^{m} \frac{R_{i}\left[1-e^{-x_{i}}\left(1+x_{i}\right)\right]^{\alpha}\left\{\ln \left[1-e^{-x_{i}}\left(1+x_{i}\right)\right]\right\}^{3}}{\left(1-\left[1-e^{-x_{i}}\left(1+x_{i}\right)\right]^{\alpha}\right)^{2}} \\
-\sum_{i=1}^{m} \frac{2 R_{i}\left[1-e^{-x_{i}}\left(1+x_{i}\right)\right]^{2 \alpha}\left\{\left[1-e^{-x_{i}}\left(1+x_{i}\right)\right]\right\}^{2}}{\left(1-\left[1-e^{-x_{i}}\left(1+x_{i}\right)\right]^{\alpha}\right)^{3}} \\
\sigma_{\alpha \alpha}=-\left(\frac{1}{L_{\alpha \alpha}}\right), \quad \tau_{\alpha}=\frac{a-1}{\alpha}-b, \quad r_{\alpha}=-\left[1-e^{-t}(1+t)\right]^{\alpha} \ln \left[1-e^{-t}(1+t)\right]
\end{gathered}
$$

and

$$
r_{\alpha \alpha}=-\left[1-e^{-t}(1+t)\right]^{\alpha}\left\{\ln \left[1-e^{-t}(1+t)\right]\right\}^{2}
$$

all the above derivatives are evaluated at the point ${ }^{\wedge} \alpha \mathrm{M}$. If $\mathrm{n}$ is sufficiently large then ${ }^{\wedge} \alpha \mathrm{BL} \rightarrow$ $\alpha^{\wedge} \mathrm{M}$.

\subsection{T-K Approximation Method}


In this subsection, we use an alternative method of Lindley's approximation to evaluate the explicit expression for the posterior expectation. This method is very useful as compared to the Lindley's and some other computational methods. This method was initially introduced by TierneyKadane (1986). A very useful compression between Lindley's and T-K method can be seen in Hawaldar and Hoosaini (2002) and Rastogi \& Tripathi (2012, 2014). To implement this method, let us consider a parametric function $\mathrm{H}(\alpha)$ and define,

$$
\begin{aligned}
& \eta(\alpha)=\frac{L(\alpha \mid \underline{\mathrm{x}})+\ln \pi(\alpha)}{n} \\
& \eta^{*}(\alpha)=\eta(\alpha)+\frac{\ln H(\alpha)}{n}
\end{aligned}
$$

3.2 T-K Approximation Method

Here, $\mathrm{L}(\alpha \mid \mathrm{x})$ is the log-likelihood function and $\pi(\alpha)$ is the prior distribution. Suppose that ${ }^{\wedge} \alpha \eta$ and $\hat{\alpha} \alpha \eta$ are the values of $\alpha$ which maximizes the above equations. Then using the method of Tierney and Kadane the posterior expectation is approximated as;

$$
\hat{H}=\sqrt{\frac{\left|\Sigma^{*}\right|}{|\Sigma|}} \exp \left[n\left\{\eta^{*}\left({\hat{\alpha_{\eta}}}_{\eta}\right)-\eta\left(\hat{\alpha_{\eta}}\right)\right\}\right]
$$

where, $|\Sigma|$ and $|\Sigma *|$ are the negative of inverse Hessian of $\eta(\alpha)$ and $\eta *(\alpha)$ respectively computed at ${ }^{\wedge} \alpha \eta$, $\hat{\alpha}\rceil *$.

Now, we derive the Bayes estimators of shape parameter $\alpha$ and reliability function $R(t)$ using this method. Therefore, in our considered case,

$$
\begin{gathered}
\eta(\alpha)=\frac{L(\alpha \mid \underline{\mathrm{x}})}{n}+\frac{(a-1) * \ln \alpha-b \alpha}{n} \\
\frac{\partial \eta}{\partial \alpha}=\frac{1}{n}\left\{\frac{\partial L}{\partial \alpha}+\frac{a-1}{\alpha}-b\right\}, \quad \frac{\partial^{2} \eta}{\partial \alpha^{2}}=\frac{1}{n}\left\{\frac{\partial^{2} L}{\partial \alpha^{2}}-\frac{a-1}{\alpha^{2}}\right\},|\Sigma|=-E\left[\frac{\partial^{2} \eta}{\partial \alpha^{2}}\right]_{\hat{\alpha}_{\eta}}^{-1}
\end{gathered}
$$

To compute the approximate Bayes estimates of $\alpha$, we take $H(\alpha)=\alpha$, then the function $\eta *(\alpha)$ will be;

$$
\eta *(\alpha)=\eta(\alpha)+\frac{\ln \alpha}{n}
$$


558 Bayesian Estimation for Exponentiated Gamma Distribution Under Progressive Type-II Censoring Using Different Approximation Techniques

$$
\frac{\partial \eta *}{\partial \alpha}=\frac{\partial \eta}{\partial \alpha}+\frac{1}{n \alpha}, \quad \frac{\partial^{2} \eta *}{\partial \alpha^{2}}=\frac{\partial^{2} \eta}{\partial \alpha^{2}}-\frac{1}{n \alpha^{2}}, \quad\left|\Sigma_{\alpha}^{*}\right|=-E\left[\frac{\partial^{2} \eta *}{\partial \alpha^{2}}\right]_{\hat{\alpha}_{\eta *}}^{-1}
$$

Thus, the desired Bayes estimate of $\alpha$ is finally obtained as;

$$
\hat{\alpha}_{T K}=\sqrt{\frac{\left|\Sigma_{\alpha}^{*}\right|}{|\Sigma|}} \exp \left[n\left\{\eta^{*}\left(\hat{\alpha_{\eta_{*}}}\right)-\eta\left(\hat{\alpha_{\eta}}\right)\right\}\right]
$$

Now, for the Bayes estimates of the reliability function $R(t), H(\alpha)=1-\left\{1-e^{-t}(1+t)\right\}^{\alpha}$, then we have

$$
\eta_{R T}^{*}(\alpha)=\eta(\alpha)+\frac{\ln H(\alpha)}{n}
$$

and

$$
\begin{gathered}
\frac{\partial \eta_{R T}^{*}}{\partial \alpha}=\frac{\partial \eta}{\partial \alpha}+\frac{H^{\prime}(\alpha)}{H(\alpha)}, \quad \frac{\partial^{2} \eta_{R T}^{*}}{\partial \alpha^{2}}=\frac{\partial^{2} \eta}{\partial \alpha^{2}}-\frac{H(\alpha) H^{\prime \prime}(\alpha)-\left[H^{\prime}(\alpha)\right]^{2}}{[H(\alpha)]^{2}} \\
\left|\Sigma_{R T}^{*}\right|=-E\left[\frac{\partial^{2} \eta *}{\partial \alpha^{2}}\right]_{\hat{\alpha}_{\eta *}}^{-1},
\end{gathered}
$$

3.3 Markov Chain Monte Carlo Method

Thus, the desired Bayes estimate of RT is obtained as;

$$
\hat{R}_{T K}=\sqrt{\frac{\left|\Sigma_{R T}^{*}\right|}{|\Sigma|}} \exp \left[n\left\{\eta^{*}\left(\hat{\alpha_{\eta_{*}}}\right)-\eta\left(\hat{\alpha_{\eta}}\right)\right\}\right]
$$

In the next subsection, we use the Markov Chain Monte Carlo method to compute the Bayes estimates for the parameter and reliability function.

\subsection{Markov Chain Monte Carlo Method}

The Markov Chain Monte Carlo method (MCMC) is one of the best technique for obtaining the Bayes estimates. This method provides the flexibility of extracting the posterior samples from its respective posterior distribution. Here, we consider the Metropolis-Hastings algorithm, to generate samples from the full conditional posterior distributions and then compute the Bayes estimates. For more details about the MCMC methods see, for example, Smith and Gelfand (1990), Hastings (1970), Upadhyaya and Gupta (2010). Therefore the marginal posterior distribution is; 


$$
\begin{aligned}
p(\alpha \mid \underline{\mathrm{x}}) & \propto \prod_{j=1}^{m}\left[x_{j}\left\{1-e^{-x_{j}}\left(1+x_{j}\right)\right\}^{\alpha-1}\left\{1-\left\{1-e^{-x_{j}}\left(1+x_{j}\right)\right\}^{\alpha}\right\}^{R_{j}}\right] \\
\alpha^{m+a-1} & \left\{e^{-\sum_{j=1}^{m} x_{j}-b \alpha}\right\}
\end{aligned}
$$

The following steps are taken into account to generate the sample from above posterior distribution.

- set the initial values of $\alpha$ say $\alpha 0$

- Set $\mathrm{k}=1$

- Generate posterior sample for $\alpha$ from (10).

- Repeat step 2 , for all $\mathrm{k}=1,2,3, \cdots \mathrm{M}$ and obtain $\alpha 1, \alpha 2, \cdots, \alpha \mathrm{M}$

- After obtaining the MCMC samples, the Bayes estimates of the parameter and reliability function under SELF are the mean of the posterior samples.

- Therefore, we have,

$$
\begin{gathered}
\hat{\alpha}_{B S}=\frac{1}{M} \sum_{k=1}^{M} \alpha_{M} \\
\hat{R}_{B S}=\frac{1}{M} \sum_{k=1}^{M}\left\{1-\left\{1-e^{-t}(1+t)\right\}^{\alpha_{k}}\right\}
\end{gathered}
$$

- The Bayesian credible interval for the parameter is obtained by using the generated MCMC samples. Thus, to construct $95 \%$ credible interval order $\alpha 1, \alpha 2, \ldots, \alpha \mathrm{M}$ as $\alpha 1<\alpha 2<\cdots<\alpha \mathrm{M}$. Then $100(1-\varphi) \%$ credible intervals of $\alpha$ is

$$
(\alpha 1, \alpha[M(1-\varphi)+1]), \cdots,(\alpha[M \varphi], \alpha M)
$$

Here $[\mathrm{x}]$ denotes the greatest integer less than or equal to $\mathrm{x}$. Then the HPD credible interval is that interval which has the shortest length.

\section{Real Data Application}


560 Bayesian Estimation for Exponentiated Gamma Distribution Under Progressive Type-II Censoring Using Different Approximation Techniques

In this section, we consider a real data set to show the applicability of the considered model. The considered real data set represent the average monthly rainfall obtained from the Information System for Management of Water Resources from the State of So Paulo, including a period of 56 years from 1947 to 2003, for the month of November., which is represented as;

Fitting of the given data set has been checked through different method of estimation using empirical cdf and qq plots. Also Likelihood ratio test has been performed to test the hypothesis H0: $\alpha=$ $1[\operatorname{Gamma}(2,1)]$ against $\mathrm{H} 1: \alpha 6=1[\mathrm{EGD}]$. Let us consider the ratio $\mathrm{T}$ as;

$$
T=\frac{\hat{L}_{H_{0}}}{\hat{L}_{H_{1}}}=\frac{1}{\alpha^{n} \prod_{i=1}^{n}\left(1-e^{-x}(1+x)\right)^{\alpha-1}}
$$

The above expression shows that the ratio $\mathrm{T}$ is less than unity i.e. $\mathrm{L}^{\wedge} \mathrm{H} 0<\mathrm{L}^{\wedge} \mathrm{H} 1$. It means that the likelihood under alternative hypothesis contains more information than the null hypothesis. Therefore, one can recommend to select exponentiated gamma distribution (EGD) instead of the other family of distribution. Further, the point estimation of the parameter and reliability function have been done by considering different variation of censoring schemes using various approximation techniques see Tables 1. Also, the interval estimates of the parameter are presented in Table 2. In order to implement MCMC algorithm in he considered real data set the convergence of chain has been acheived by tuning process. In this context the trace and density plot based on 10000 MCMC samples are given in figure 2, which shows that the generated sample for the considered proposal distribution are well mixed and cover true value of the parameter. 


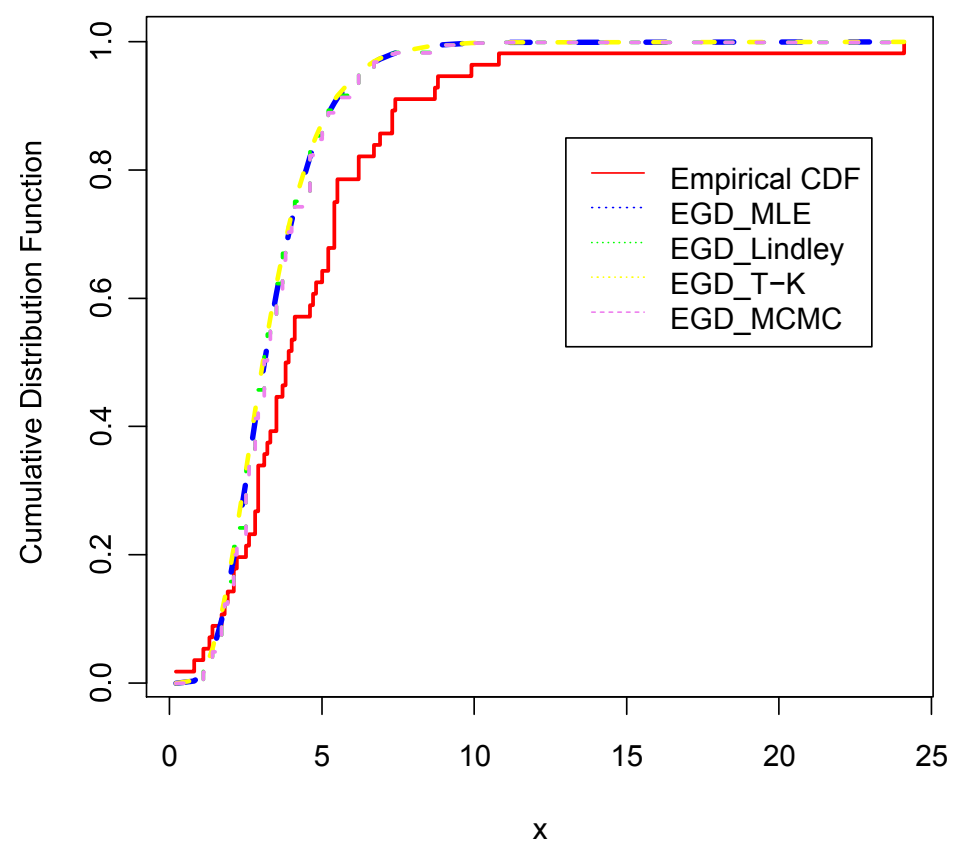

Figure 1: Empirical CDF plot for the real data.
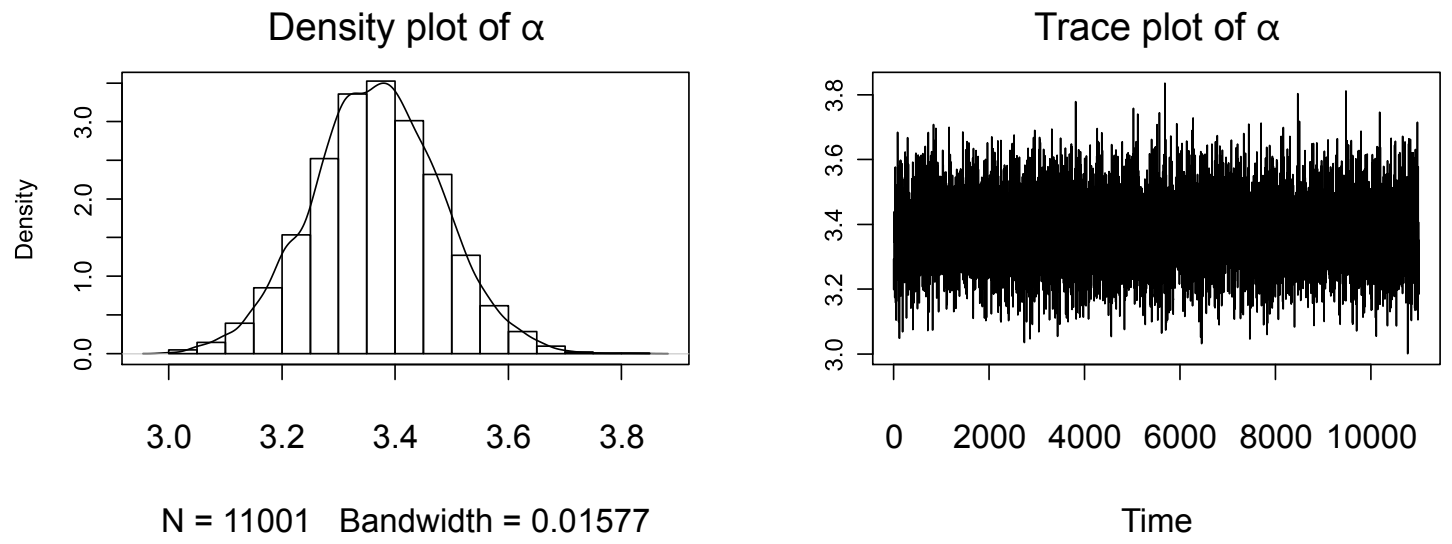

Figure 2: Convergence chain plot for the real data set.

\section{Numerical Study}

In previous sections, we have obtained the mathematical expression for maximum likelihood and Bayes estimators for unknown parameter and reliability function under progressive type-II censoring. 
562 Bayesian Estimation for Exponentiated Gamma Distribution Under Progressive Type-II Censoring Using Different Approximation Techniques

In this section, we compare the performances of these estimators numerically using Monte Carlo simulations. For comparison purpose, we have generated a random number from exponentiated gamma distribution for $\alpha=2$. The progressive type-II censored samples are generated using Balakrishanan and Shandhu algorithm. Two value of sample size $n=20,30$ are taken and corresponding effective sample sizes are chosen in such a way that the observed samples are $50 \%$, $60 \%$ and $80 \%$ are censored. For each effective sample sizes four different censoring schemes are chosen as the removals occurs at (i); each stages (ii): at initial stages, (iii): last stages and (iv): mid points. The mean square error criterion is considered for compression of the estimators. MLE of the parameter is obtained by using non-linear maximization technique and corresponding reliability function is obtained by using invariance property. The Bayes estimates of the parameter and reliability function are obtained under independent gamma prior using squared error loss function. The Bayes estimates are computed with the help of Lindley's, T-K and Markov Chain Monte Carlo methods. The value of hyper parameters are assumed as $\mathrm{a}=4, \mathrm{~b}=2$. The reliability estimates are evaluated for $t=3$, where, actual reliability $R(t)=0.3586$. Further, Bayes estimators are also derived with respect to a non-informative prior distribution where the values of each hyper parameters are assigned as zero. From the extensive study of simulation, we see that the estimates obtained by Lindley's approximation method have less variability as compared to the estimates obtained by rest of other two methods when informative prior is considered. Performances of the estimators are quite similar in case of non-informative prior. The average estimates and corresponding MSE are reported in Tables 3-6. From Tables we observed that;

- The mean square error of all estimators are decreases when the sample size $n$ and effective sample size $\mathrm{m}$ are increases.

- The mean square error of the Bayes estimators are smaller than the mean square error of maximum likelihood estimators in the case of informative prior but in other hand the mean square error of the all estimators are approximately same when non-informative prior is taken.

- Under three approximation techniques, the mean square error of Bayes estimates of the parameter under informative prior following patterns is noticed;

$$
\operatorname{MSEs}\left(\operatorname{Lindley}^{0} S\right)<\operatorname{MSEs}(T-K) \leq \operatorname{MSEs}(M C M C)<\operatorname{MSES}(M L E)
$$

In other hand, the MSEs of the estimates of the parameter are almost same in the case of noninformative prior.

- Under three approximation techniques, the mean square error of Bayes estimates of the reliability function obtained under informative as well as non-informative we noticed that;

$$
\operatorname{MSES}\left(\operatorname{Lindley}^{0} s\right)<\operatorname{MSEs}(T-K)<\operatorname{MSES}(M C M C)<\operatorname{MSES}(M L E)
$$


- The Length of the $95 \%$ Bayes intervals is much smaller than the asymptotic confidence intervals.

- The credible length of interval in case of informative prior is smaller than the credible length of intervals obtained under non-informative prior in all considered cases.

Table 1: Estimates of the parameter and reliability function for the considered real data set.

\begin{tabular}{|c|c|c|c|c|c|c|c|c|c|c|}
\hline $\mathrm{n}$ & $\mathrm{m}$ & Scheme & $\hat{\alpha}_{M L}$ & $\hat{\alpha}_{L}$ & $\hat{\alpha}_{T K}$ & $\hat{\alpha}_{M C}$ & $\hat{R}_{M L}$ & $\hat{R}_{L}$ & $\hat{R}_{T K}$ & $\hat{R}_{M C}$ \\
\hline \multirow{10}{*}{56} & 56 & $0 * 56$ & 3.4094 & 3.4094 & 3.4095 & 3.4060 & 0.5310 & 0.5286 & 0.5286 & 0.5305 \\
\hline & \multirow{3}{*}{46} & $10 * 1,0 * 45$ & 4.2709 & 4.2709 & 4.2711 & 4.2490 & 0.6127 & 0.6089 & 0.6089 & 0.6094 \\
\hline & & $0 * 45,10 * 1$ & 2.8265 & 2.8265 & 2.8266 & 2.8201 & 0.4662 & 0.4639 & 0.4639 & 0.4649 \\
\hline & & $0^{*} 18,1^{*} 10,0^{*} 18$ & 2.9964 & 2.9964 & 2.9966 & 2.9855 & 0.4860 & 0.4835 & 0.4835 & 0.4841 \\
\hline & \multirow{3}{*}{36} & $5^{*} 4,0^{*} 32$ & 5.4532 & 5.4532 & 5.4536 & 5.4295 & 0.7021 & 0.6960 & 0.6961 & 0.6993 \\
\hline & & $0 * 32,5 * 4$ & 2.2468 & 2.2468 & 2.2469 & 2.2436 & 0.3928 & 0.3907 & 0.3908 & 0.3923 \\
\hline & & $0 * 8,1 * 20,0 * 8$ & 2.6313 & 2.6313 & 2.6315 & 2.6294 & 0.4425 & 0.4399 & 0.4399 & 0.4421 \\
\hline & \multirow{3}{*}{26} & $5^{*} 6,0^{*} 20$ & 2.7700 & 2.7700 & 2.7704 & 2.7631 & 0.4595 & 0.4555 & 0.4556 & 0.4583 \\
\hline & & $0 * 20,5 * 6$ & 2.8982 & 2.8982 & 2.8986 & 2.8907 & 0.4746 & 0.4704 & 0.4705 & 0.4734 \\
\hline & & $0 * 8,3 * 10,0 * 8$ & 2.5428 & 2.5428 & 2.5431 & 2.5348 & 0.4315 & 0.4280 & 0.4281 & 0.4302 \\
\hline
\end{tabular}

Table 2: 95\% confidence intervals for the parameter of considered real data set.

\begin{tabular}{cccccccc}
\hline & $\mathrm{m}$ & Scheme $\hat{\alpha_{L}}$ & $\hat{\alpha_{U}}$ & Length & $\hat{\alpha_{L^{B}}}{\hat{\alpha_{U}}}^{B}$ Length & \\
\hline 56 & $0^{*} 56$ & 2.5163 & 4.3024 & 1.7861 & 3.1886 & 3.6265 & 0.4379 \\
\cline { 2 - 8 } 46 & $10^{*} 1,0^{*} 45$ & 3.0365 & 5.5052 & 2.4687 & 3.4920 & 4.9694 & 1.4773 \\
& $0^{*} 45,10^{*} 1$ & 2.0096 & 3.6434 & 1.6338 & 2.4470 & 3.2017 & 0.7548 \\
& $0^{*} 18,1^{*} 10,0^{*} 18$ & 2.1304 & 3.8624 & 1.7320 & 2.5795 & 3.4058 & 0.8263 \\
\cline { 2 - 8 } 36 & $5^{*} 4,0^{*} 32$ & 3.6716 & 7.2348 & 3.5631 & 4.6217 & 6.2198 & 1.5981 \\
& $0^{*} 32,5^{*} 4$ & 1.5127 & 2.9808 & 1.4680 & 2.0935 & 2.3886 & 0.2951 \\
& $0^{*} 8,1^{*} 20,0^{*} 8$ & 1.7717 & 3.4910 & 1.7193 & 2.4248 & 2.8347 & 0.4099 \\
\cline { 2 - 8 } 26 & $5^{*} 6,0^{*} 20$ & 1.7052 & 3.8349 & 2.1298 & 2.4585 & 3.0736 & 0.6152 \\
& $0^{*} 20,5^{*} 6$ & 1.7840 & 4.0123 & 2.2283 & 2.5455 & 3.2154 & 0.6699 \\
& $0^{*} 8,3^{*} 10,0^{*} 8$ & 1.5653 & 3.5204 & 1.9551 & 2.2675 & 2.7881 & 0.5206
\end{tabular}


564 Bayesian Estimation for Exponentiated Gamma Distribution Under Progressive Type-II Censoring Using Different Approximation Techniques

Table 3: Average estimates and MSEs of the parameter for different variation of $n$, $\mathrm{m}$ and RI under informative prior when, $\alpha=2$.

\begin{tabular}{|c|c|c|c|c|c|c|c|c|c|c|}
\hline \multirow{2}{*}{$\mathrm{n}$} & \multirow{2}{*}{$\mathbf{m}$} & \multirow{2}{*}{ RI } & \multicolumn{2}{|c|}{ MLE } & \multicolumn{6}{|c|}{ Bayes } \\
\hline & & & $\hat{\alpha}_{M L}$ & MSE & $\hat{\alpha}_{L}$ & MSE & $\hat{\alpha}_{T K}$ & MSE & $\hat{\alpha}_{M C}$ & MSE \\
\hline \multirow{13}{*}{20} & 20 & $0 * 20$ & 2.0960 & 0.2285 & 2.0539 & 0.1258 & 2.0652 & 0.1453 & 1.9449 & 0.1310 \\
\hline & \multirow{4}{*}{10} & $1 * 10$ & 2.1026 & 0.2673 & 2.0593 & 0.1331 & 2.0719 & 0.1610 & 1.9531 & 0.1446 \\
\hline & & $2 * 5,0 * 5$ & 2.1392 & 0.3130 & 2.0852 & 0.1462 & 2.1012 & 0.1824 & 1.9757 & 0.1587 \\
\hline & & $0 * 5,2 * 5$ & 2.0978 & 0.2663 & 2.0539 & 0.1387 & 2.0662 & 0.1642 & 1.9553 & 0.1504 \\
\hline & & $0 * 4,5 * 2,0 * 4$ & 2.1222 & 0.2856 & 2.0732 & 0.1411 & 2.0870 & 0.1711 & 1.9762 & 0.1531 \\
\hline & \multirow{4}{*}{12} & $1 * 8,0 * 4$ & 2.1065 & 0.2590 & 2.0630 & 0.1330 & 2.0753 & 0.1583 & 1.9758 & 0.1543 \\
\hline & & $0 * 4,1 * 8$ & 2.1159 & 0.2713 & 2.0671 & 0.1385 & 2.0805 & 0.1654 & 1.9830 & 0.1543 \\
\hline & & $0 * 4,2 * 4,0 * 4$ & 2.1072 & 0.2497 & 2.0633 & 0.1327 & 2.0752 & 0.1555 & 1.9815 & 0.1517 \\
\hline & & $0 * 5,4 * 2,0 * 5$ & 2.1071 & 0.2449 & 2.0638 & 0.1307 & 2.0755 & 0.1529 & 1.9827 & 0.1497 \\
\hline & \multirow{4}{*}{16} & $1 * 4,0 * 12$ & 2.1089 & 0.2833 & 2.0609 & 0.1406 & 2.0749 & 0.1702 & 2.0050 & 0.1815 \\
\hline & & $0 * 12,1 * 4$ & 2.0830 & 0.2406 & 2.0425 & 0.1295 & 2.0538 & 0.1510 & 1.9810 & 0.1593 \\
\hline & & $4,0^{*} 15$ & 2.0959 & 0.2736 & 2.0513 & 0.1343 & 2.0650 & 0.1637 & 1.9906 & 0.1773 \\
\hline & & $0 * 6,1 * 4,0 * 6$ & 2.1233 & 0.3118 & 2.0681 & 0.1462 & 2.0839 & 0.1820 & 2.0147 & 0.1825 \\
\hline \multirow{13}{*}{30} & 30 & $0 * 30$ & 2.0853 & 0.1785 & 2.0620 & 0.1222 & 2.0664 & 0.1302 & 1.9538 & 0.1106 \\
\hline & \multirow{4}{*}{15} & $1 * 15$ & 2.0729 & 0.1633 & 2.0559 & 0.1115 & 2.0592 & 0.1189 & 2.0036 & 0.1249 \\
\hline & & $3 * 5,0 * 10$ & 2.1040 & 0.2260 & 2.0819 & 0.1411 & 2.0866 & 0.1552 & 2.0290 & 0.1644 \\
\hline & & $0 * 10,3 * 5$ & 2.1005 & 0.1782 & 2.0766 & 0.1202 & 2.0810 & 0.1287 & 2.0285 & 0.1302 \\
\hline & & $0 * 5,3 * 5,0 * 5$ & 2.0518 & 0.1636 & 2.0377 & 0.1116 & 2.0406 & 0.1190 & 1.9885 & 0.1275 \\
\hline & \multirow{4}{*}{18} & $1 * 12,0^{*} 6$ & 2.0903 & 0.1803 & 2.0694 & 0.1211 & 2.0735 & 0.1298 & 2.0292 & 0.1401 \\
\hline & & $0 * 6,1 * 12$ & 2.0457 & 0.1525 & 2.0309 & 0.1076 & 2.0339 & 0.1136 & 1.9875 & 0.1234 \\
\hline & & $0^{*} 6,2^{*} 6,0^{*} 6$ & 2.0652 & 0.1547 & 2.0484 & 0.1075 & 2.0516 & 0.1139 & 2.0081 & 0.1237 \\
\hline & & $0 * 3,1 * 12,0 * 3$ & 2.0946 & 0.1743 & 2.0725 & 0.1191 & 2.0767 & 0.1269 & 2.0334 & 0.1350 \\
\hline & \multirow{4}{*}{24} & $2^{*} 3,0^{*} 21$ & 2.0590 & 0.1681 & 2.0428 & 0.1126 & 2.0464 & 0.1209 & 2.0168 & 0.1413 \\
\hline & & $0 * 9,1 * 6,0 * 9$ & 2.0684 & 0.1676 & 2.0487 & 0.1151 & 2.0526 & 0.1226 & 2.0237 & 0.1388 \\
\hline & & $0 * 18,1 * 6$ & 2.0792 & 0.1514 & 2.0586 & 0.1058 & 2.0625 & 0.1120 & 2.0310 & 0.1241 \\
\hline & & $3,0^{*} 22,3$ & 2.0788 & 0.1717 & 2.0577 & 0.1158 & 2.0620 & 0.1241 & 2.0307 & 0.1402 \\
\hline
\end{tabular}

Table 4: Average estimates and MSEs of the reliability function $\mathrm{R}(\mathrm{t})$ for different variation of $\mathrm{n}, \mathrm{m}$ and RI under informative prior, when $t=3$.

\begin{tabular}{|c|c|c|c|c|c|c|c|c|c|c|}
\hline \multirow{2}{*}{$\mathbf{n}$} & \multirow{2}{*}{$\mathbf{m}$} & \multirow{2}{*}{ RI } & \multicolumn{2}{|c|}{ MLE } & \multicolumn{6}{|c|}{ Bayes } \\
\hline & & & $\hat{R}(t)_{M L}$ & MSE & $\hat{R}(t)_{L}$ & MSE & $\hat{R}(t)_{T K}$ & MSE & $\hat{R}(t)_{M C}$ & MSE \\
\hline \multirow{13}{*}{20} & 20 & $0 * 20$ & 0.3688 & 0.0041 & 0.3612 & 0.0023 & 0.3629 & 0.0026 & 0.3482 & 0.0027 \\
\hline & \multirow{4}{*}{10} & $1 * 10$ & 0.3692 & 0.0047 & 0.3615 & 0.0024 & 0.3634 & 0.0029 & 0.3493 & 0.0030 \\
\hline & & $2 * 5,0 * 5$ & 0.3738 & 0.0054 & 0.3648 & 0.0026 & 0.3671 & 0.0032 & 0.3522 & 0.0032 \\
\hline & & $0 * 5,2 * 5$ & 0.3686 & 0.0047 & 0.3609 & 0.0026 & 0.3627 & 0.0029 & 0.3496 & 0.0031 \\
\hline & & $0 * 4,5 * 2,0 * 4$ & 0.3718 & 0.0049 & 0.3635 & 0.0025 & 0.3655 & 0.0030 & 0.3525 & 0.0030 \\
\hline & \multirow{4}{*}{12} & $1 * 8,0^{*} 4$ & 0.3699 & 0.0046 & 0.3620 & 0.0024 & 0.3639 & 0.0028 & 0.3524 & 0.0031 \\
\hline & & $0 * 4,1 * 8$ & 0.3711 & 0.0047 & 0.3628 & 0.0025 & 0.3647 & 0.0029 & 0.3535 & 0.0030 \\
\hline & & $0 * 4,2 * 4,0 * 4$ & 0.3701 & 0.0045 & 0.3622 & 0.0024 & 0.3641 & 0.0028 & 0.3533 & 0.0030 \\
\hline & & $0 * 5,4 * 2,0 * 5$ & 0.3702 & 0.0044 & 0.3623 & 0.0024 & 0.3642 & 0.0027 & 0.3535 & 0.0030 \\
\hline & \multirow{4}{*}{16} & $1 * 4,0 * 12$ & 0.3699 & 0.0049 & 0.3618 & 0.0025 & 0.3638 & 0.0030 & 0.3562 & 0.0035 \\
\hline & & $0 * 12,1 * 4$ & 0.3668 & 0.0043 & 0.3595 & 0.0024 & 0.3612 & 0.0027 & 0.3532 & 0.0031 \\
\hline & & $4,0 * 15$ & 0.3682 & 0.0048 & 0.3603 & 0.0025 & 0.3623 & 0.0029 & 0.3542 & 0.0035 \\
\hline & & $0 * 6,1 * 4,0 * 6$ & 0.3717 & 0.0050 & 0.3630 & 0.0027 & 0.3650 & 0.0031 & 0.3576 & 0.0035 \\
\hline \multirow{13}{*}{30} & 30 & $0 * 30$ & 0.3681 & 0.0032 & 0.3634 & 0.0022 & 0.3642 & 0.0023 & 0.3498 & 0.0022 \\
\hline & \multirow{4}{*}{15} & $1 * 15$ & 0.3665 & 0.0030 & 0.3625 & 0.0021 & 0.3631 & 0.0022 & 0.3571 & 0.0025 \\
\hline & & $3 * 5,0 * 10$ & 0.3700 & 0.0040 & 0.3654 & 0.0025 & 0.3662 & 0.0027 & 0.3600 & 0.0031 \\
\hline & & $0 * 10,3 * 5$ & 0.3703 & 0.0032 & 0.3654 & 0.0022 & 0.3662 & 0.0023 & 0.3606 & 0.0025 \\
\hline & & $0^{*} 5,3 * 5,0^{*} 5$ & 0.3635 & 0.0030 & 0.3600 & 0.0021 & 0.3606 & 0.0022 & 0.3549 & 0.0025 \\
\hline & \multirow{4}{*}{18} & $1 * 12,0 * 6$ & 0.3687 & 0.0033 & 0.3642 & 0.0022 & 0.3650 & 0.0024 & 0.3605 & 0.0027 \\
\hline & & $0 * 6,1 * 12$ & 0.3628 & 0.0029 & 0.3592 & 0.0020 & 0.3597 & 0.0021 & 0.3548 & 0.0024 \\
\hline & & $0^{*} 6,2 * 6,0 * 6$ & 0.3655 & 0.0029 & 0.3616 & 0.0020 & 0.3622 & 0.0021 & 0.3577 & 0.0024 \\
\hline & & $0 * 3,1 * 12,0 * 3$ & 0.3695 & 0.0032 & 0.3648 & 0.0022 & 0.3656 & 0.0023 & 0.3612 & 0.0026 \\
\hline & \multirow{4}{*}{24} & $2 * 3,0^{*} 21$ & 0.3645 & 0.0031 & 0.3606 & 0.0021 & 0.3613 & 0.0022 & 0.3587 & 0.0027 \\
\hline & & $0 * 9,1 * 6,0 * 9$ & 0.3658 & 0.0030 & 0.3616 & 0.0021 & 0.3623 & 0.0022 & 0.3597 & 0.0026 \\
\hline & & $0 * 18,1 * 6$ & 0.3676 & 0.0028 & 0.3632 & 0.0019 & 0.3639 & 0.0021 & 0.3610 & 0.0024 \\
\hline & & $3,0 * 22,3$ & 0.3672 & 0.0031 & 0.3627 & 0.0021 & 0.3635 & 0.0023 & 0.3607 & 0.0027 \\
\hline
\end{tabular}


Table 5: Average estimates and MSEs of the parameter for different variation of $n$, $\mathrm{m}$ and RI under non-informative prior when $\alpha=2$.

\begin{tabular}{|c|c|c|c|c|c|c|c|c|c|c|}
\hline \multirow{2}{*}{$\mathrm{n}$} & \multirow{2}{*}{$\mathrm{m}$} & \multirow{2}{*}{ RI } & \multicolumn{2}{|c|}{ MLE } & \multicolumn{6}{|c|}{ Bayes } \\
\hline & & & $\hat{\alpha}_{M L}$ & MSE & $\hat{\alpha}_{L}$ & MSE & $\hat{\alpha}_{T K}$ & MSE & $\hat{\alpha}_{M C}$ & MSE \\
\hline & 20 & $0 * 20$ & 2.0960 & 0.2285 & 2.1050 & 0.2347 & 2.1138 & 0.2534 & 2.0969 & 0.2456 \\
\hline \multirow{13}{*}{20} & \multirow{4}{*}{10} & $1 * 10$ & 2.1026 & 0.2673 & 2.1106 & 0.2832 & 2.1203 & 0.3053 & 1.7148 & 0.1994 \\
\hline & & $2 * 5,0^{*} 5$ & 2.1392 & 0.3130 & 2.0930 & 0.2611 & 2.1081 & 0.2982 & 1.7962 & 0.1927 \\
\hline & & $0 * 5,2 * 5$ & 2.0978 & 0.2663 & 2.0924 & 0.2481 & 2.1066 & 0.2815 & 1.6604 & 0.2053 \\
\hline & & $0 * 4,5 * 2,0 * 4$ & 2.1222 & 0.2856 & 2.1090 & 0.2729 & 2.1119 & 0.2793 & 1.7363 & 0.1850 \\
\hline & \multirow{4}{*}{12} & $1 * 8,0 * 4$ & 2.1065 & 0.2590 & 2.1184 & 0.2504 & 2.1230 & 0.2590 & 1.8493 & 0.1634 \\
\hline & & $0 * 4,1 * 8$ & 2.1159 & 0.2713 & 2.1191 & 0.2256 & 2.1216 & 0.2302 & 1.7673 & 0.1523 \\
\hline & & $0 * 4,2 * 4,0 * 4$ & 2.1072 & 0.2497 & 2.1004 & 0.2644 & 2.1072 & 0.2785 & 1.7989 & 0.1782 \\
\hline & & $0 * 5,4 * 2,0 * 5$ & 2.1071 & 0.2449 & 2.1288 & 0.2805 & 2.1390 & 0.3069 & 1.8264 & 0.1805 \\
\hline & \multirow{4}{*}{16} & $1 * 4,0 * 12$ & 2.1089 & 0.2833 & 2.0987 & 0.2631 & 2.1181 & 0.3098 & 2.0179 & 0.2532 \\
\hline & & $0 * 12,1 * 4$ & 2.0830 & 0.2406 & 2.0896 & 0.2338 & 2.0967 & 0.2497 & 1.8883 & 0.1701 \\
\hline & & $4,0 * 15$ & 2.0959 & 0.2736 & 2.0941 & 0.2633 & 2.0951 & 0.2637 & 2.0267 & 0.2332 \\
\hline & & $0 * 6,1 * 4,0 * 6$ & 2.1233 & 0.3118 & 2.1006 & 0.2287 & 2.1104 & 0.2523 & 1.9429 & 0.1779 \\
\hline & 30 & $0 * 30$ & 2.0853 & 0.1785 & 2.0440 & 0.1403 & 2.0442 & 0.1403 & 2.0325 & 0.1395 \\
\hline \multirow{12}{*}{30} & \multirow{4}{*}{15} & $1 * 15$ & 2.0729 & 0.1633 & 2.0634 & 0.1702 & 2.0659 & 0.1753 & 1.7709 & 0.1306 \\
\hline & & $3 * 5,0 * 10$ & 2.1040 & 0.2260 & 2.0553 & 0.1873 & 2.0579 & 0.1919 & 1.7591 & 0.1709 \\
\hline & & $0 * 10,3 * 5$ & 2.1005 & 0.1782 & 2.0597 & 0.1424 & 2.0641 & 0.1517 & 1.9276 & 0.1019 \\
\hline & & $0 * 5,3 * 5,0 * 5$ & 2.0518 & 0.1636 & 2.0649 & 0.1618 & 2.0651 & 0.1618 & 1.8711 & 0.1081 \\
\hline & \multirow{4}{*}{18} & $1 * 12,0 * 6$ & 2.0903 & 0.1803 & 2.0777 & 0.1866 & 2.0780 & 0.1867 & 1.8768 & 0.1278 \\
\hline & & $0^{*} 6,1^{*} 12$ & 2.0457 & 0.1525 & 2.0821 & 0.1660 & 2.0845 & 0.1714 & 1.8207 & 0.1166 \\
\hline & & $0 * 6,2 * 6,0 * 6$ & 2.0652 & 0.1547 & 2.0797 & 0.1745 & 2.0799 & 0.1746 & 1.8507 & 0.1178 \\
\hline & & $0^{*} 3,1^{*} 12,0^{*} 3$ & 2.0946 & 0.1743 & 2.0708 & 0.1598 & 2.0731 & 0.1640 & 1.8407 & 0.1142 \\
\hline & \multirow{4}{*}{24} & $2 * 3,0 * 21$ & 2.0590 & 0.1681 & 2.0839 & 0.1745 & 2.0862 & 0.1785 & 2.0001 & 0.1554 \\
\hline & & $0 * 9,1 * 6,0 * 9$ & 2.0684 & 0.1676 & 2.0588 & 0.1630 & 2.0590 & 0.1630 & 1.8290 & 0.1452 \\
\hline & & $0 * 18,1 * 6$ & 2.0792 & 0.1514 & 2.0634 & 0.1467 & 2.0656 & 0.1511 & 1.7669 & 0.1500 \\
\hline & & $3,0 * 22,3$ & 2.0788 & 0.1717 & 2.0823 & 0.1831 & 2.0846 & 0.1872 & 1.8808 & 0.1514 \\
\hline
\end{tabular}

Table 6: Average estimates and MSEs of the reliability function $\mathrm{R}(\mathrm{t})$ for different variation of $\mathrm{n}, \mathrm{m}$ and RI under non-informative prior, when $t=3$.

\begin{tabular}{|c|c|c|c|c|c|c|c|c|c|c|}
\hline \multirow[b]{2}{*}{$\mathbf{n}$} & \multirow[b]{2}{*}{$\mathrm{m}$} & \multirow{2}{*}{$\mathrm{RI}$} & \multicolumn{2}{|c|}{ MLE } & \multicolumn{6}{|c|}{ Bayes } \\
\hline & & & $\hat{R}(t)_{M L}$ & MSE & $\hat{R}(t)_{L}$ & MSE & $\hat{R}(t)_{T K}$ & MSE & $\hat{R}(t)_{M C}$ & MSE \\
\hline & 20 & $0 * 20$ & 0.3688 & 0.0041 & 0.3675 & 0.0042 & 0.3676 & 0.0043 & 0.3658 & 0.0042 \\
\hline \multirow{13}{*}{20} & \multirow{4}{*}{10} & $1 * 10$ & 0.3692 & 0.0047 & 0.3673 & 0.0051 & 0.3674 & 0.0051 & 0.3124 & 0.0046 \\
\hline & & $2 * 5,0 * 5$ & 0.3738 & 0.0054 & 0.3654 & 0.0049 & 0.3656 & 0.0049 & 0.3239 & 0.0042 \\
\hline & & $0 * 5,2 * 5$ & 0.3686 & 0.0047 & 0.3660 & 0.0046 & 0.3661 & 0.0046 & 0.3047 & 0.0048 \\
\hline & & $0 * 4,5 * 2,0 * 4$ & 0.3718 & 0.0049 & 0.3666 & 0.0046 & 0.3667 & 0.0047 & 0.3157 & 0.0042 \\
\hline & \multirow{4}{*}{12} & $1^{*} 8,0^{*} 4$ & 0.3699 & 0.0046 & 0.3688 & 0.0039 & 0.3689 & 0.0039 & 0.3210 & 0.0035 \\
\hline & & $0 * 4,1 * 8$ & 0.3711 & 0.0047 & 0.3683 & 0.0043 & 0.3685 & 0.0043 & 0.3323 & 0.0035 \\
\hline & & $0 * 4,2 * 4,0 * 4$ & 0.3701 & 0.0045 & 0.3660 & 0.0046 & 0.3662 & 0.0047 & 0.3250 & 0.0039 \\
\hline & & $0 * 5,4 * 2,0 * 5$ & 0.3702 & 0.0044 & 0.3701 & 0.0050 & 0.3702 & 0.0050 & 0.3288 & 0.0038 \\
\hline & \multirow{4}{*}{16} & $1 * 4,0 * 12$ & 0.3699 & 0.0049 & 0.3670 & 0.0049 & 0.3672 & 0.0049 & 0.3553 & 0.0044 \\
\hline & & $0 * 12,1 * 4$ & 0.3668 & 0.0043 & 0.3652 & 0.0042 & 0.3653 & 0.0042 & 0.3383 & 0.0034 \\
\hline & & $4,0 * 15$ & 0.3682 & 0.0048 & 0.3641 & 0.0046 & 0.3643 & 0.0046 & 0.3567 & 0.0043 \\
\hline & & $0 * 6,1 * 4,0 * 6$ & 0.3717 & 0.0050 & 0.3670 & 0.0042 & 0.3671 & 0.0042 & 0.3459 & 0.0034 \\
\hline & 30 & $0 * 30$ & 0.3681 & 0.0032 & 0.3606 & 0.0026 & 0.3606 & 0.0026 & 0.3594 & 0.0026 \\
\hline \multirow{12}{*}{30} & \multirow{4}{*}{15} & $1 * 15$ & 0.3665 & 0.0030 & 0.3629 & 0.0031 & 0.3629 & 0.0031 & 0.3227 & 0.0029 \\
\hline & & $3 * 5,0 * 10$ & 0.3700 & 0.0040 & 0.3612 & 0.0034 & 0.3613 & 0.0034 & 0.3194 & 0.0038 \\
\hline & & $0 * 10,3 * 5$ & 0.3703 & 0.0032 & 0.3631 & 0.0027 & 0.3632 & 0.0027 & 0.3463 & 0.0021 \\
\hline & & $0 * 5,3 * 5,0 * 5$ & 0.3635 & 0.0030 & 0.3630 & 0.0029 & 0.3631 & 0.0029 & 0.3377 & 0.0023 \\
\hline & \multirow{4}{*}{18} & $1 * 12,0^{*} 6$ & 0.3687 & 0.0033 & 0.3644 & 0.0033 & 0.3645 & 0.0033 & 0.3379 & 0.0027 \\
\hline & & $0 * 6,1 * 12$ & 0.3628 & 0.0029 & 0.3657 & 0.0030 & 0.3658 & 0.0030 & 0.3302 & 0.0026 \\
\hline & & $0 * 6,2 * 6,0 * 6$ & 0.3655 & 0.0029 & 0.3650 & 0.0031 & 0.3650 & 0.0031 & 0.3344 & 0.0025 \\
\hline & & $0 * 3,1 * 12,0 * 3$ & 0.3695 & 0.0032 & 0.3642 & 0.0029 & 0.3642 & 0.0029 & 0.3330 & 0.0025 \\
\hline & \multirow{4}{*}{24} & $2 * 3,0^{*} 21$ & 0.3645 & 0.0031 & 0.3656 & 0.0031 & 0.3657 & 0.0031 & 0.3541 & 0.0029 \\
\hline & & $0 * 9,1 * 6,0 * 9$ & 0.3658 & 0.0030 & 0.3623 & 0.0029 & 0.3623 & 0.0029 & 0.3301 & 0.0031 \\
\hline & & $0 * 18,1 * 6$ & 0.3676 & 0.0028 & 0.3634 & 0.0027 & 0.3635 & 0.0027 & 0.3213 & 0.0034 \\
\hline & & $3,0 * 22,3$ & 0.3672 & 0.0031 & 0.3654 & 0.0033 & 0.3654 & 0.0033 & 0.3373 & 0.0032 \\
\hline
\end{tabular}


566 Bayesian Estimation for Exponentiated Gamma Distribution Under Progressive Type-II Censoring Using Different Approximation Techniques

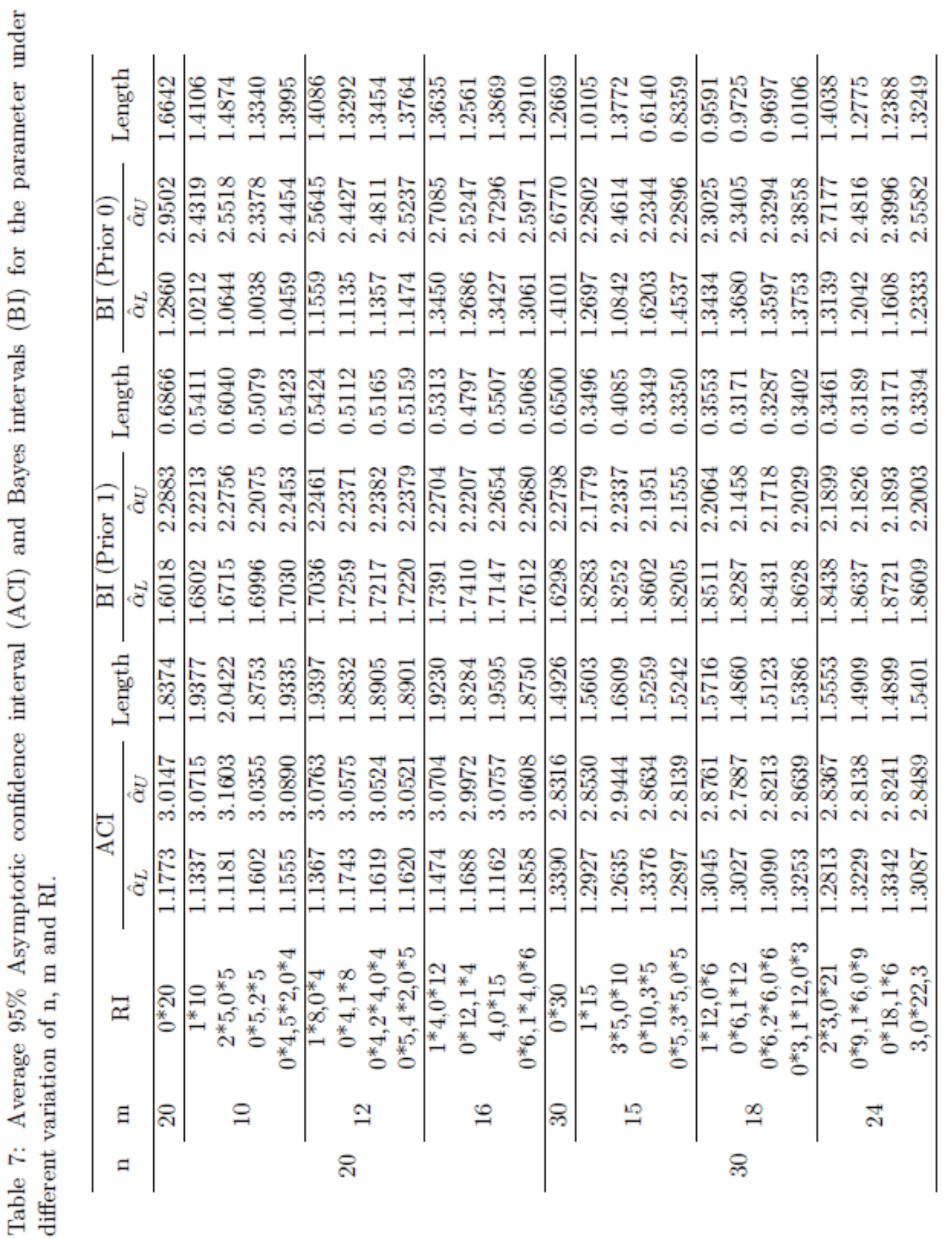




\section{Conclusions}

In this paper, we proposed the Bayes estimation of the unknown parameter and reliability function of the exponentiated gamma distribution under progressive type-II censored data. The proposed estimators are compared along with maximum likelihood estimators using Monte Carlo simulations. MLEs is obtained by using non-linear maximization method. The Bayes estimates of the parameter and reliability function are computed under the assumption of independent gamma prior using Lindley's, $\mathrm{T}-\mathrm{K}$ and MCMC techniques under squared error loss function. It is observed that the approximation techniques works very well and we noticed that the performances of Bayes estimators obtained under informative prior using Lindley's approximation have smaller mean square error as compared to the rest of the methods while the MSEs of the Bayes estimators are quite similar under T-K and MCMC methods. The performances of the estimators are almost same in the case on non-informative prior in all approximation methods.

\section{Acknowledgements}

The authors are thankful to the Editor and the referee for careful reading and comments which greatly improved the quality of the manuscript.

\section{References}

[1]. R. C. Gupta, R. D. Gupta and P. L. Gupta, Modeling Failure Time Data by Lehman Alternatives, Commun. Statist.-Theory, Meth., vol. 27, no. 4, (1998), pp. 887-904.

[2]. S. K. Singh, U. Singh and D. Kumar,Bayesian Estimation of The Exponentiated Gamma Parameter and Reliability Function Under Asymmetric Loss Function, REVSTAT-Statistical Journal, vol. 9, no. 3, (2011), pp. 247-260.

[3]. A. I. Shawky and R. A. Bakoban (2008). Bayesian and Non-Bayesian Estimations on the Exponentiated Gamma Distribution. Applied Mathematical Sciences, vol. 2, no. 51, pp. 2521 2530.

[4]. I. Shawky and R. A. Bakoban (2009). Order Statistics from Exponentiated Gamma Distribution and Associated Inference. Int. J. Contemp. Math. Sciences, vol. 4, no. 2, pp. 71 91.

[5]. I. Shawky and R. A. Bakoban (2009) On Finite Mixture of Two-Component Exponentiated Gamma Distribution. Journal of Applied Sciences Research, vol. 5, no. 10, pp. 1351-1369.

[6]. Ghanizadeh, H. Pazira and R. Lotfi (2011). Classical Estimations of the Exponentiated Gamma Distribution Parameters with Presence of K Outliers. Australian Journal of Basic and Applied Sciences, vol. 5, no. 3, pp. 571-579.

[7]. R. U. Khan and D. Kumar (2011). Lower Generalized Order Statistics from Exponentiated Gamma Distribution and Its Characterization. Prob. Stat Forum, vol. 4, pp. 25-38.

[8]. D. V. Lindley (1980). Approximate Bayes method. Trabajos de estadistica, Vol. 31, 223-237.

[9]. Kadane, J. B. and Lazar, N. A. Method and criteria for model selection, Journal of the American Statistical Association, Vol. 99, pp. 279290, 2004. 
568 Bayesian Estimation for Exponentiated Gamma Distribution Under Progressive Type-II Censoring Using Different Approximation Techniques

[10]. A. E. Gelfand and A. F. M. Smith (1990). Sampling-Based Approaches to Calculating Marginal Densities. Journal of the American Statistical Association, Vol. 85, No. 410. pp. 398-409.

[11]. S. K. Upadhyay and A. Gupta (2010). A Bayes Analysis of Modified Weibull Distribution via Markov Chain, Monte Carlo Simulation. Journal. of Statistical Computation and Simulation, 80 (3), 241-254.

[12]. A.C. Cohen (1965). Maximum likelihood estimation in the Weibull distribution based on complete and censored samples. Technometrics, 7,579-588.

[13]. N. Balakrishnan, R. Aggarwala (2000). Progressive Censoring, Theory, Methods and Applications, Birkhauser, Boston, 2000.

[14]. N. Balakrishnan, A. Hossain (2007). Inference for the type II generalized logistic distribution under progressive type II censoring. J. Stat. Comput.Simul. 77 (12), 1013-1031.

[15]. N. Balakrishnan, R. A. Sandhu (1995), A simple simulation algorithm for generating progressively type II censored samples, Am. Stat. 49 (2), 229-230.

[16]. H. Krishna and Kapil Kumar (2011). Reliability estimation in Lindley distribution with progressively type II right censored sample. Mathematics and Computers in Simulation 82, 281-294.

[17]. H. Krishna and Kapil Kumar (2011). Reliability estimation in generalized inverted exponential distribution with progressively type II censored sample. Journal of Stat. Comput. and Simulation, DOI:10.1080/00949655.2011.647027.

[18]. R.D. Gupta and D. Kundu (2001). Exponentiated exponential distribution: An alternative to gamma and Weibull distributions. Biomet. J. 43(1), pp. 117-130.

[19]. Pradhan and D. Kundu (2009). On progressively censored generalized exponential distribution. Test 18,pp. 497-515.

[20]. H. Krishna and M. Malik (2011). Reliability estimation in Maxwell distribution with progressively type-II censored data, preprint. J. Statist. Comput. Simul., Available at http://dx.doi.org/10.1080/00949655.2010.550291.

[21]. N. Feroze and M. Aslam (2012). Bayesian Analysis of Exponentiated Gamma Distribution under Type II Censored Samples, International Journal of Advanced Science and Technology Vol. 49.

[22]. W. K. Hastings (1970). Monte Carlo Sampling Methods Using Markov Chains and Their Applications. Biometrika, Vol. 57, No. 1. 97-109. 